\title{
Correction to: Morphometric analysis of Chattian-Early Aquitanian Miogypsinidae from Iraq and their stratigraphic distribution in the Arabian Tethys
}

\section{Qahtan A. M. Al Nuaimy ${ }^{1}$}

Published online: 15 January 2018

(C) Saudi Society for Geosciences 2018

\section{Correction to: Arab J Geosci (2017) 10:542}

https://doi.org/10.1007/s12517-017-3311-7

The original version of this article, unfortunately, contained errors.

Corrections in Figures and Plates were incorrectly carried out. Given in this article is the correct list of images.

The original article was corrected.

The online version of the original article can be found at https://doi.org/ 10.1007/s12517-017-3311-7

Qahtan A. M. Al Nuaimy

qahtaniraqi@yahoo.com

1 Technical College, Northern Technical University, Kirkuk, Iraq 
Fig. 1 Location of the studied wells and outcrop. Source:Google Earth (www.googleearth.com)

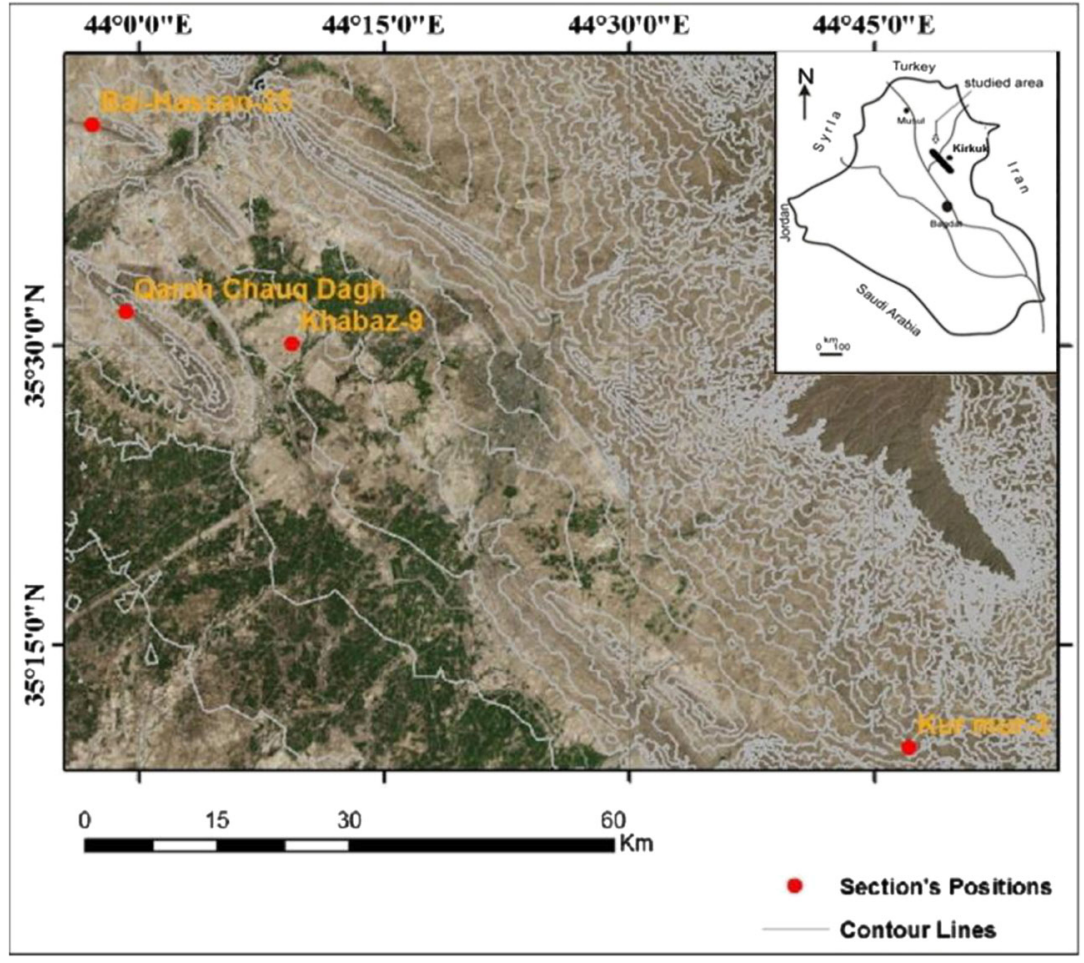




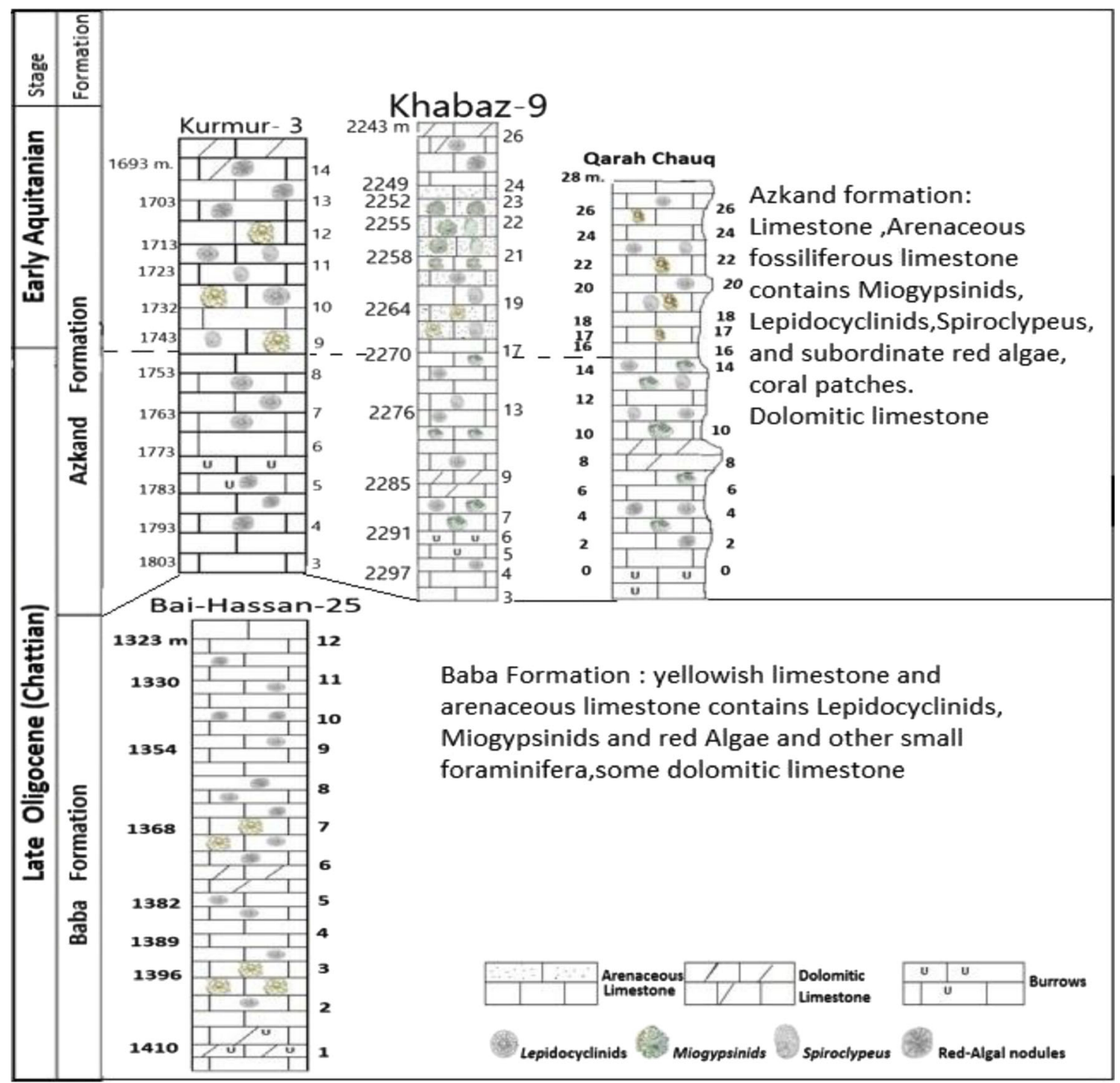

Fig. 2 General stratigraphy of the Oligocene-Miocene sediment sequence (Baba and Azkand Formation) from Khabaz-9, Kurmur-3 and Bai-Hassan-25 boreholes and the Qarah Chauq Dagh section 


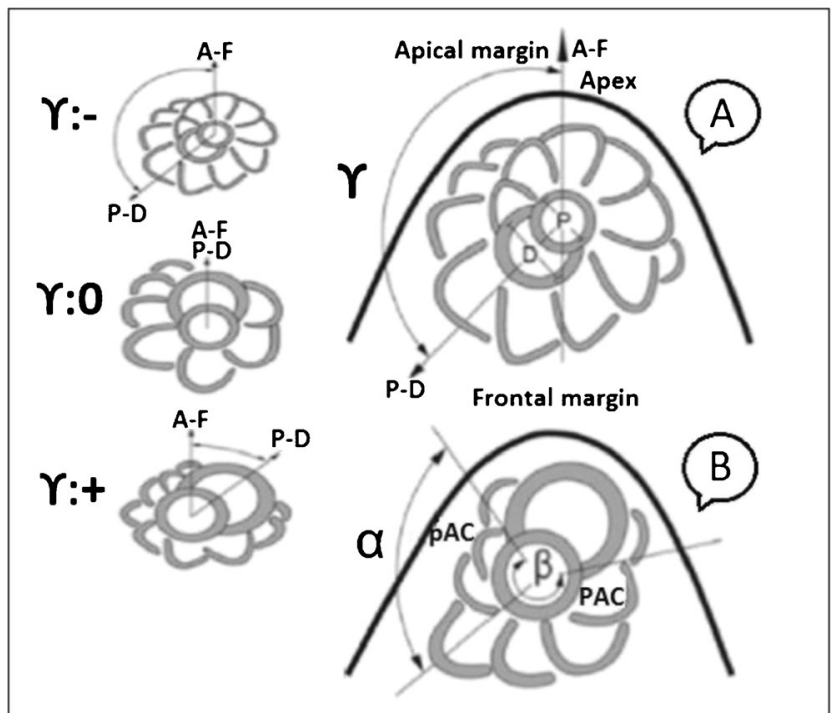

Fig. 3 Schematic drawing showing the methods of counting and measuring the internal features in horizontally sectioned embryonicnepionic stages of the megalospheric form (most parameters are explained in Table 1). a Single-spiralled Miogypsinids (A-F apicalfrontal axis, $\mathrm{P}-\mathrm{D}$ embryonic axis, $\mathrm{X}=12 ; \mathrm{Y}=5$, and $\gamma^{\circ}=$ negative). $\mathrm{b}$ Miogypsinids with two primary auxiliary chamberlets (PAC). $\gamma^{\circ}=$ positive (Amato and Drooger 1969; Drooger 1952, 1963) 


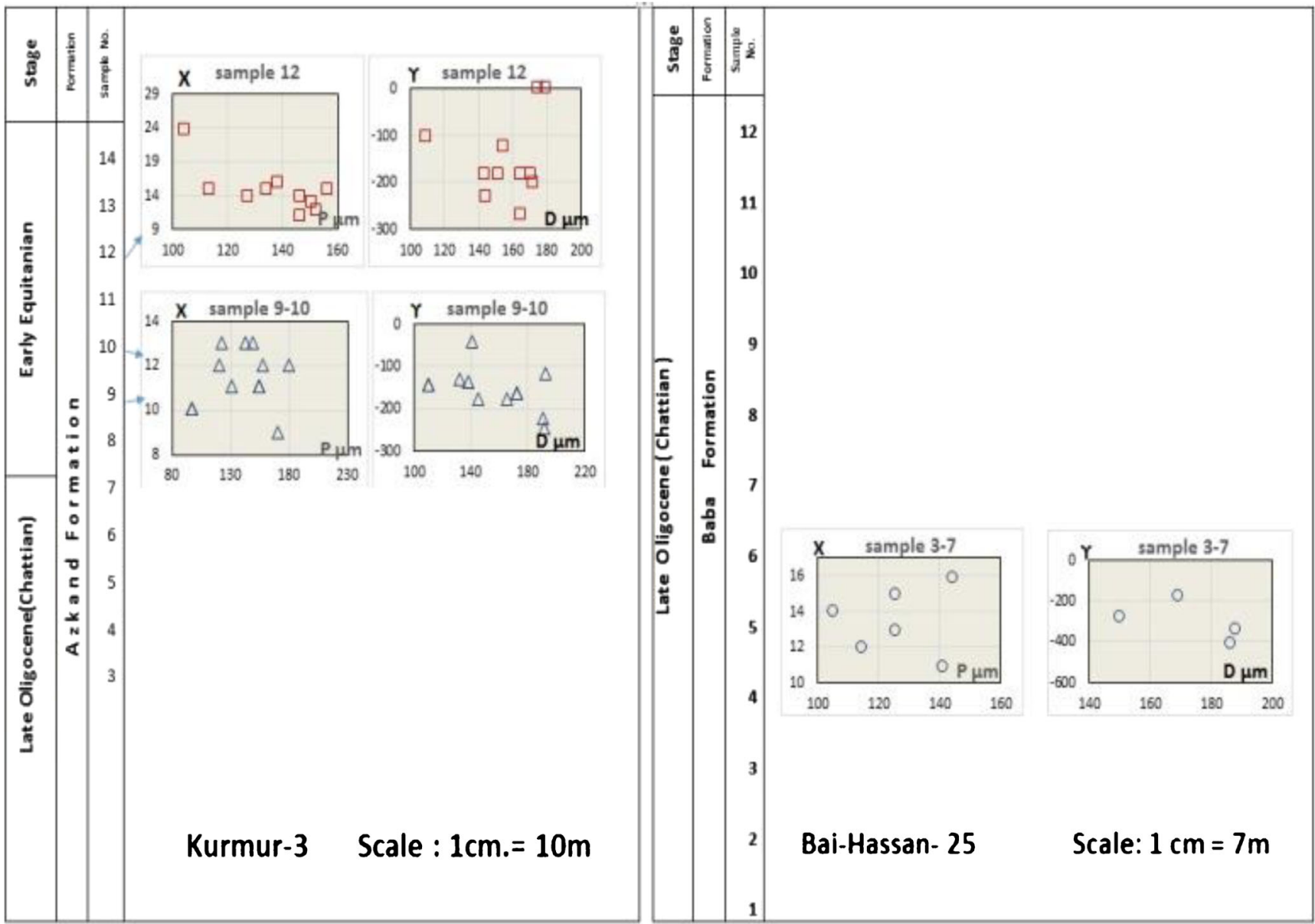

Fig. 4 Scatter diagrams of $\mathrm{P}-\mathrm{X}$ values on the left and $\mathrm{D}-\gamma$ values on the right in Miogypsinids assemblages from Kurmur well-3 of the Azkand Formation and Bia-Hassan well-25 of the Baba Formation 

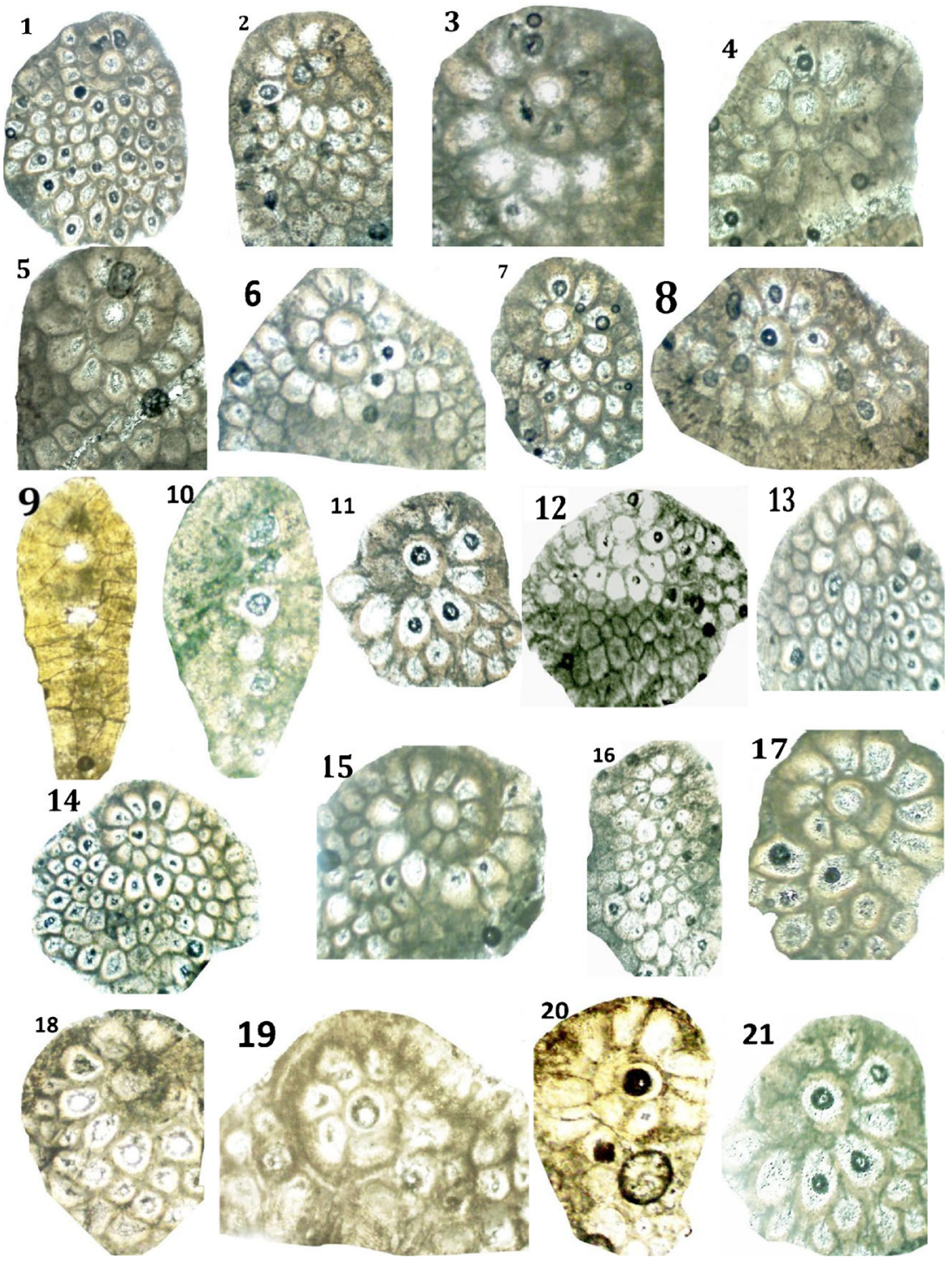

Plate 1 All A-forms. Magnification, × 40. 1-17: Miogypsina gunteri Cole. Khabaz well-9, early lower Aquitanian, depth 2255 m. 1-8: Equatorial sections. M/Khabaz 22. 1 (2-1), 2 (3), 3 (9-2), 4 (5-4), 5 (5), 6 (11-6), 7 (12-1), 8 (15-1). 9-10: Axial sections. M/Khabaz 22. 9 (2-1), 10 (8-1). 11-17: Equatorial sections. M/Khabaz 22. 11 (19-2), 12
(17-3), 13 (18-1), 14 (18-3), 15 (18-5), 16 (6), 17 (25-1). 18-21: Miogypsina ex. interc. Tani Drooger-gunteri Cole, Khabaz well-9, lower Aquitanian, Depth 2252 m. Equatorial sections. M/Khabaz 23. 18 (3), 19 (6), 20 (3-3), 21 (19-2) 


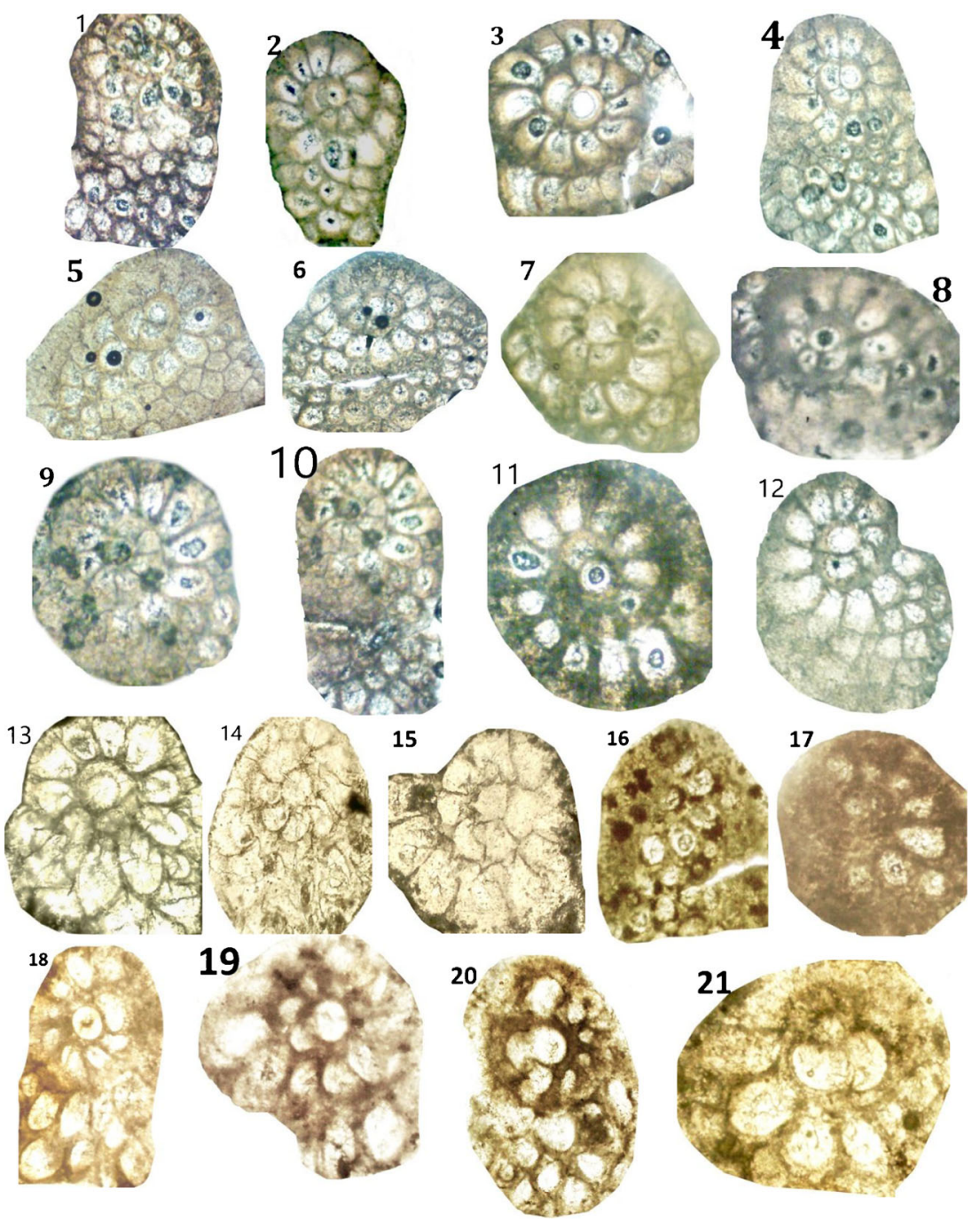

Plate 2 All A-forms. Magnification, × 40. 1-12: Miogypsina ex. interc. Gunteri Cole-basraensis Bronnimann, Khabaz well-9, early lower Aquitanian, depth $2258 \mathrm{~m}$., equatorial section. M/Khabaz 21. 1 (2-2), 2 (3-1), 3 (3-2), 4 (6), 5 (6-1), 6 (25-1), 7 (8), 8 (14-1), 9 (14-2), 10 (14 3), 11 (6), 12 (24). 13-15: Miogypsina basraensis Bronnimann, BiaHassan 25, late Oligocene (Chattian). Equatorial section. M/Bia-
Hassan. 13 (3-1), depth 1396m. 14 (3-2), depth 1396m. 15 (6-3), depth 1375 m. 16-21: Miogypsinoides sivasensis Özcan and Less, 2009, Qarah Chauq Dagh section, early lower Aquitanian. equatorial section. $16 \mathrm{M} / \mathrm{Q}$ arah Chauq 12-1, $17 \mathrm{M} / \mathrm{Q}$ arah Chauq 12, $18 \mathrm{M} / \mathrm{Qarah}$ Chauq 12-2, 19 M/Qarah Chauq 12-3, 20 M/Qarah Chauq 17 C, 21M/ Qarah Chauq $20 \mathrm{~d}$ 

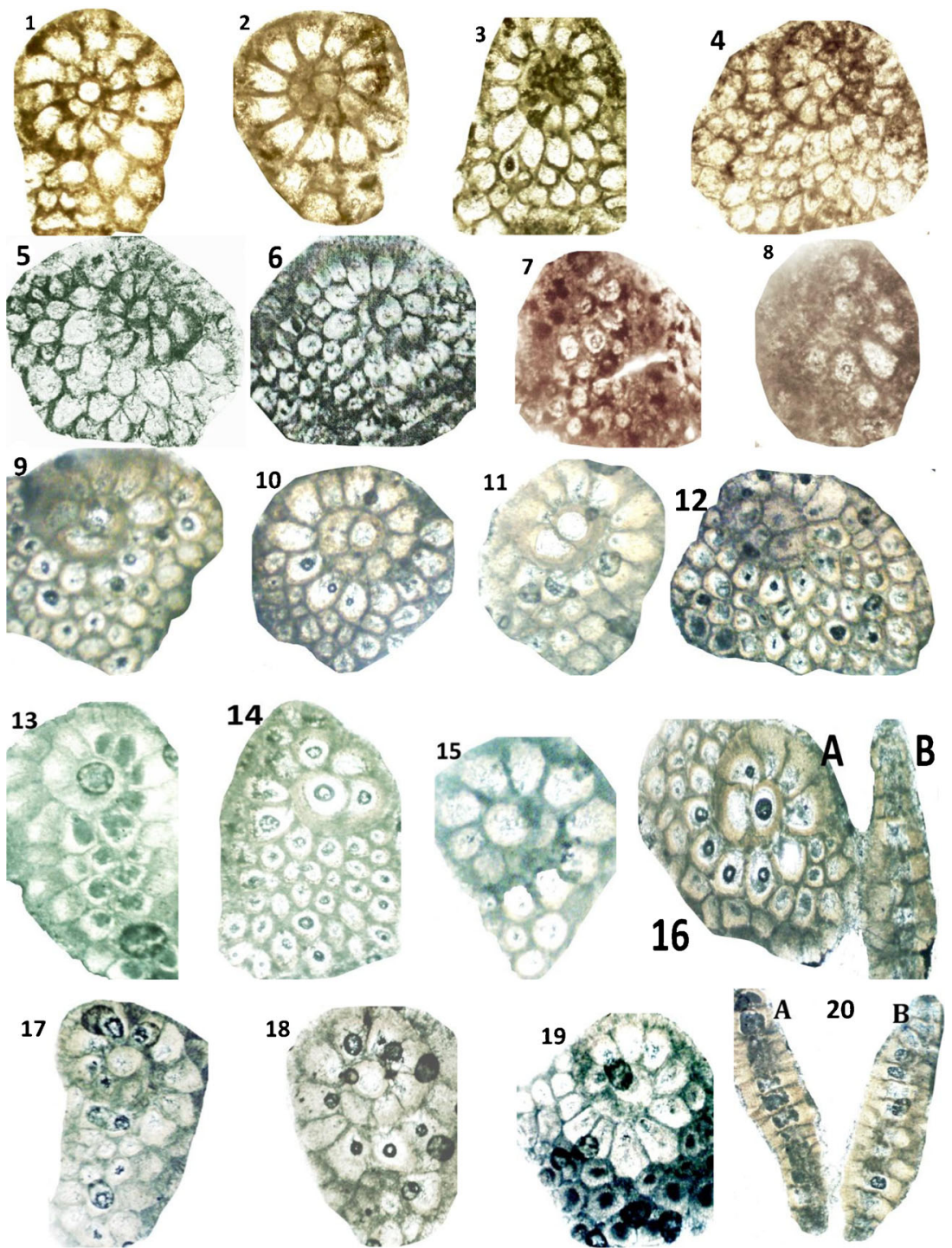

Plate 3 All A-forms. Magnification, $\times$ 40. 1-6: Miogypsinoides formosensis Yabe and Hanzawa 1928, Qarah Chauq Dagh section. Late Oligocene (Chattian), equatorial section. $1 \mathrm{Ms} /$ Qarah Chauq 3C-3, $2 \mathrm{Ms} /$ Qarah Chauq 3C-9, 3Ms/Qarah Chauq 3E-1, 4Ms/Qarah Chauq 3L-1, 5 Ms/Khabaz 7, 6 Ms/Khabaz 17. 7-8: Miogypsinoides bantamensis (Tan Sin Hok) .Khabaz well-9, early lower Aquitanian, equatorial section.Depth 2264 m. 7 Ms/Khabaz 19-2, 8 Ms/Khabaz 19-7. 9-20: Miogypsinoides dehaartii, Khabaz well-9, early lower Aquitanian. 9-12

Equatorial section. Depth 2258m., 9 Ms/Khabaz 21-17-2, 10Ms/Khabaz 21-21-1, $11 \mathrm{Ms} / \mathrm{Khabaz} 21-13,12 \mathrm{Ms} / \mathrm{Khabaz} 22-19-2.13-15$ Equatorial section. Depth 2255 m., 13 Ms/Khabaz 22-1-1, 14 Ms/Khabaz 22-6-1, 15- Ms/Khabaz 22-11-4. 16 A. Equatorial section. Ms/Khabaz 22-11-4. B. Axial section, depth 2255 m. 17-19 Equatorial section. Depth 2255 m., $17 \mathrm{Ms} / \mathrm{Khabaz} 22-14-5,18 \mathrm{Ms} / \mathrm{Khabaz} 22-8-1$, 19 Ms/Khabaz 2211-3. $20 \mathrm{Ms} /$ Khabaz 22-19; A — axial section, B — oblique axial section 


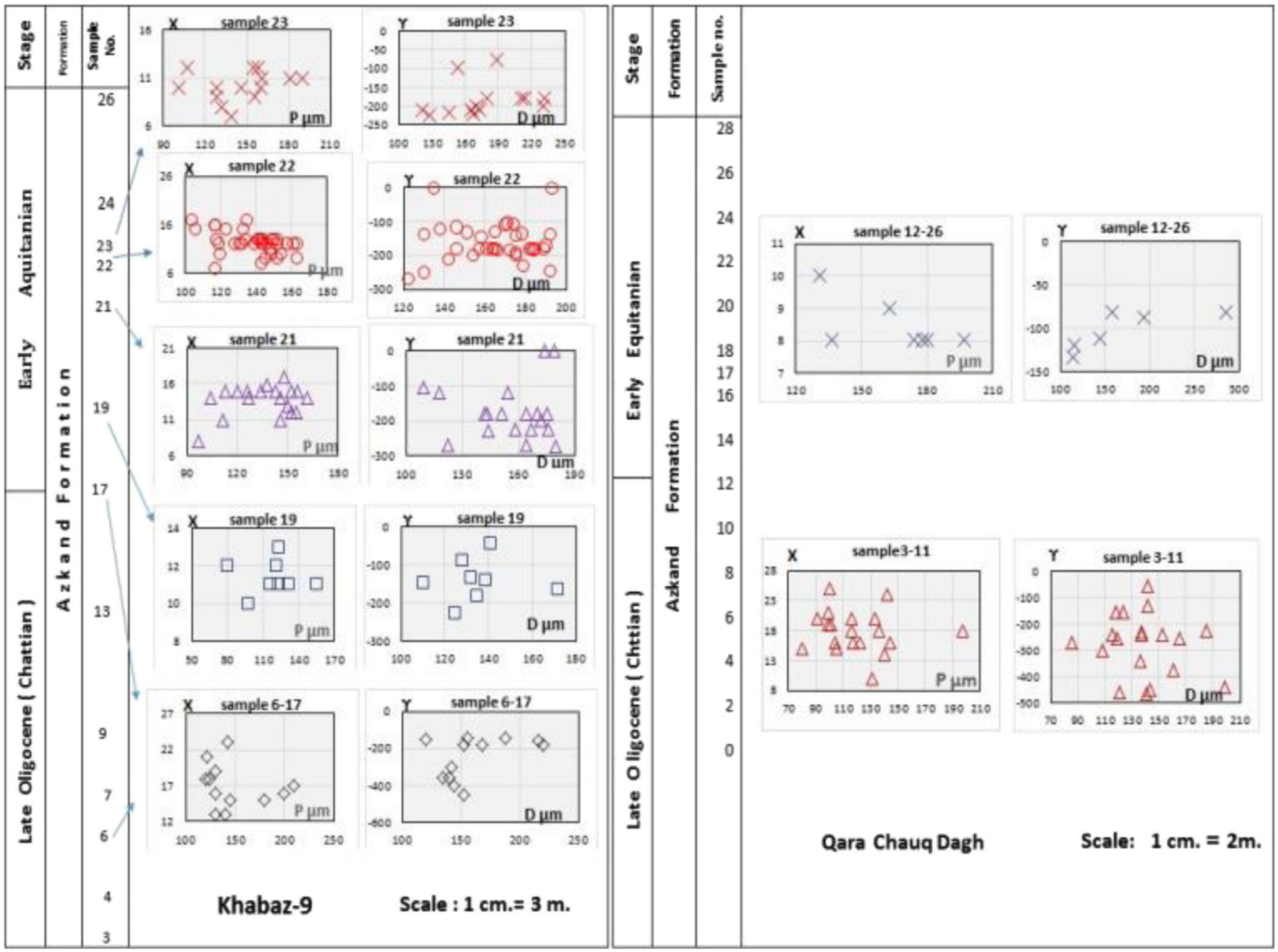

Fig. 5 Scatter diagrams of $\mathrm{P}-\mathrm{X}$ values (on the left) and $\mathrm{D}-\Upsilon$ values (on the right) in Miogypsinids assemblages from Khabaz well-9 and Qara Chauq Dagh section of the Azkand Formation
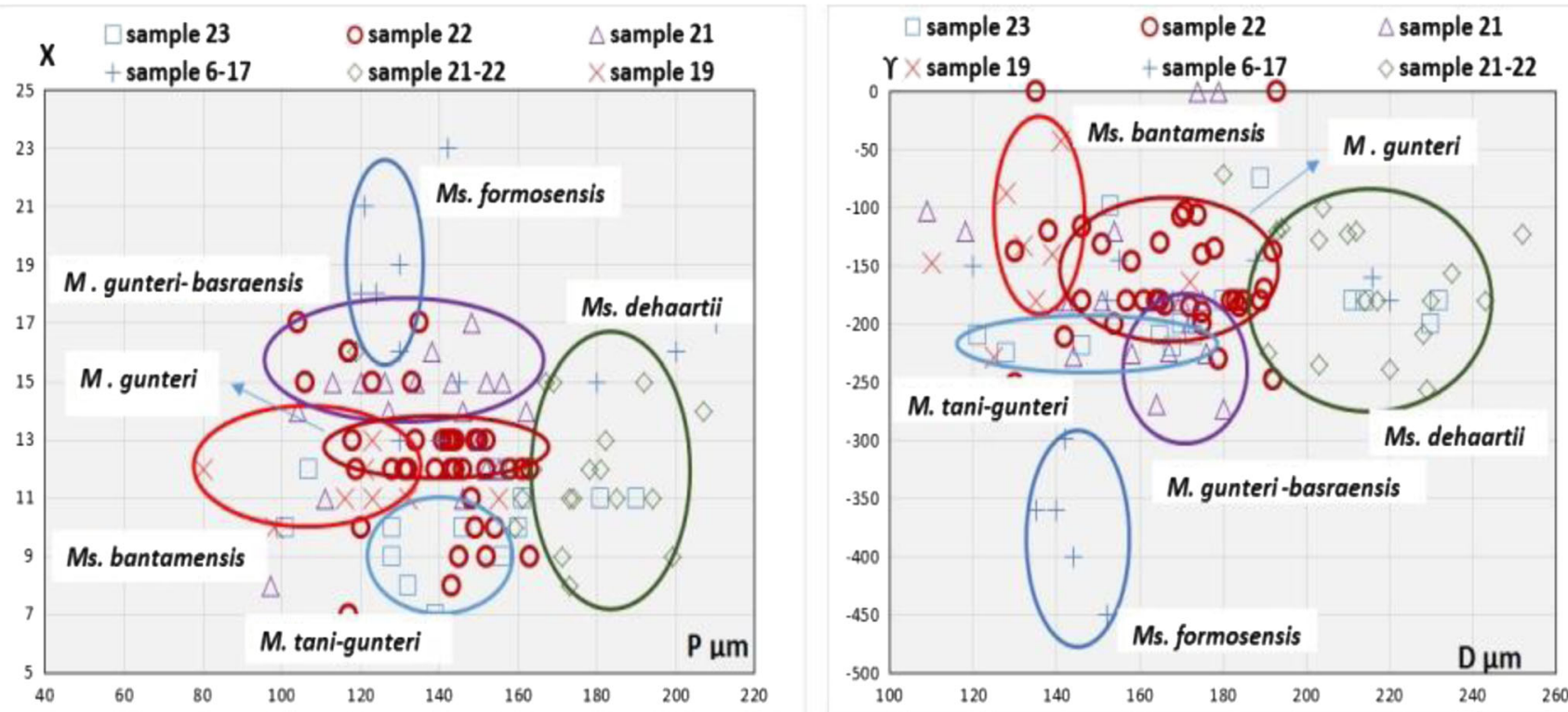

Fig. 6 Scatter diagrams of $\mathrm{P}-\mathrm{X}$ values (on the left) and $\mathrm{D}-\Upsilon$ values (on the right) in Miogypsinids assemblages from Khabaz well-9 of the Azkand Formation (M Miogypsina, Ms Miogypsinoides) 


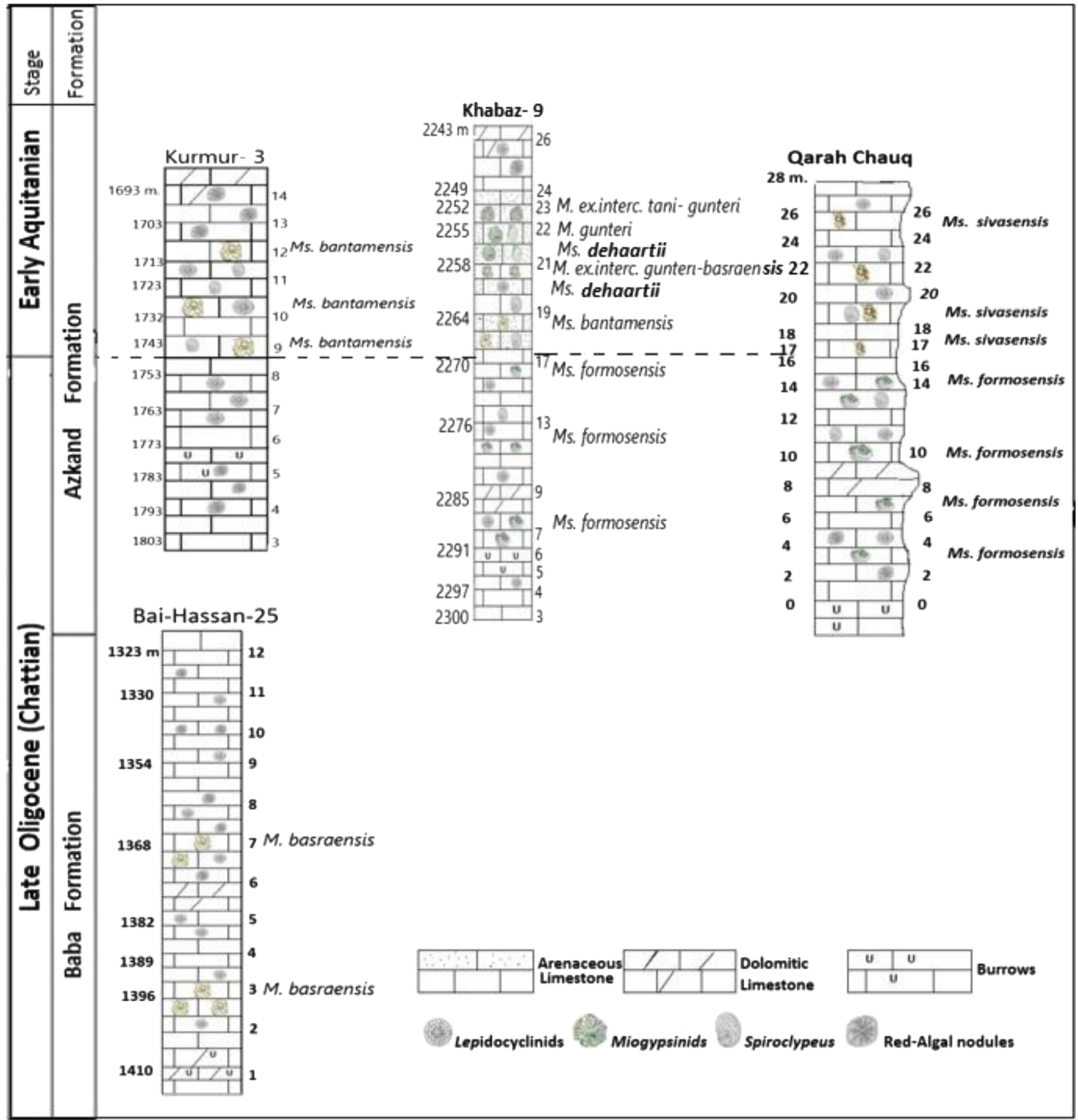

Fig. 7 Scatter diagrams of $\mathrm{P}-\mathrm{X}$ values (on the left) and $\mathrm{D}-\Upsilon$ values (on the right) in Miogypsinids assemblages from Khabaz well-9 of the Azkand Formation (M Miogypsina, Ms Miogypsinoides) 
Fig. 8 Correlation of stratigraphic sections based on the

evolutionary stages ofOligoceneEarly Miocene Miogypsinids and Lepidocyclinid assemblages

(Nephrolepidina) in northern Iraq (no. 1 refers to the Miogypsina embryon and no. 2 refers to the Miogypsinoides embryon).

Nephrolepidina data are from

Ghafor and Muhammed (2005)

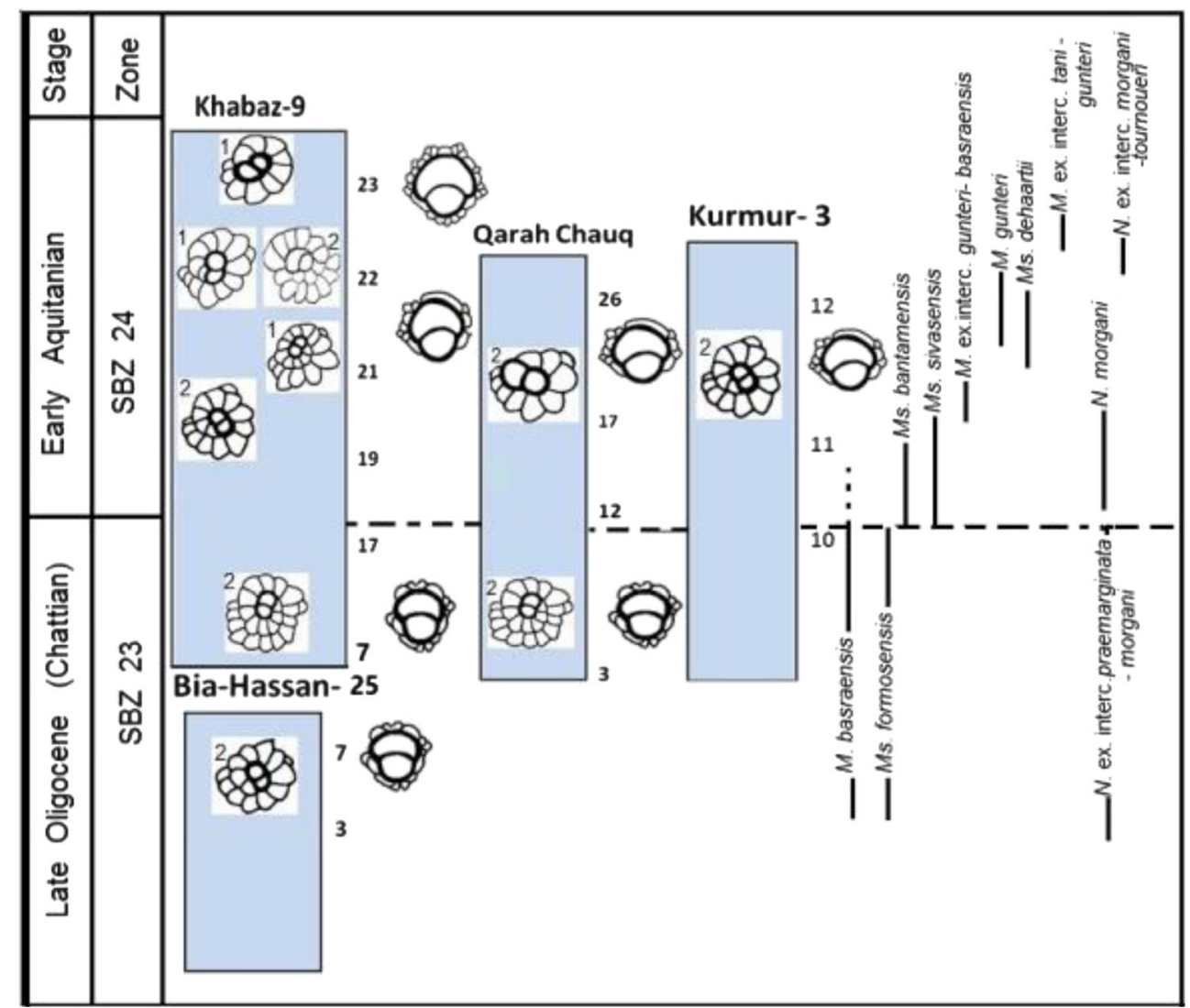

